This item was submitted to Loughborough's Research Repository by the author.

Items in Figshare are protected by copyright, with all rights reserved, unless otherwise indicated.

\title{
Eco-design tool to support the use of renewable polymers within packaging applications
}

PLEASE CITE THE PUBLISHED VERSION

http://dx.doi.org/10.1007/978-3-642-19692-8

PUBLISHER

(C) Springer

VERSION

AM (Accepted Manuscript)

LICENCE

CC BY-NC-ND 4.0

\section{REPOSITORY RECORD}

Colwill, James, Shahin Rahimifard, and Allen J. Clegg. 2019. "Eco-design Tool to Support the Use of Renewable Polymers Within Packaging Applications”. figshare. https://hdl.handle.net/2134/13303. 
This item was submitted to Loughborough's Institutional Repository (https://dspace.lboro.ac.uk/) by the author and is made available under the following Creative Commons Licence conditions.

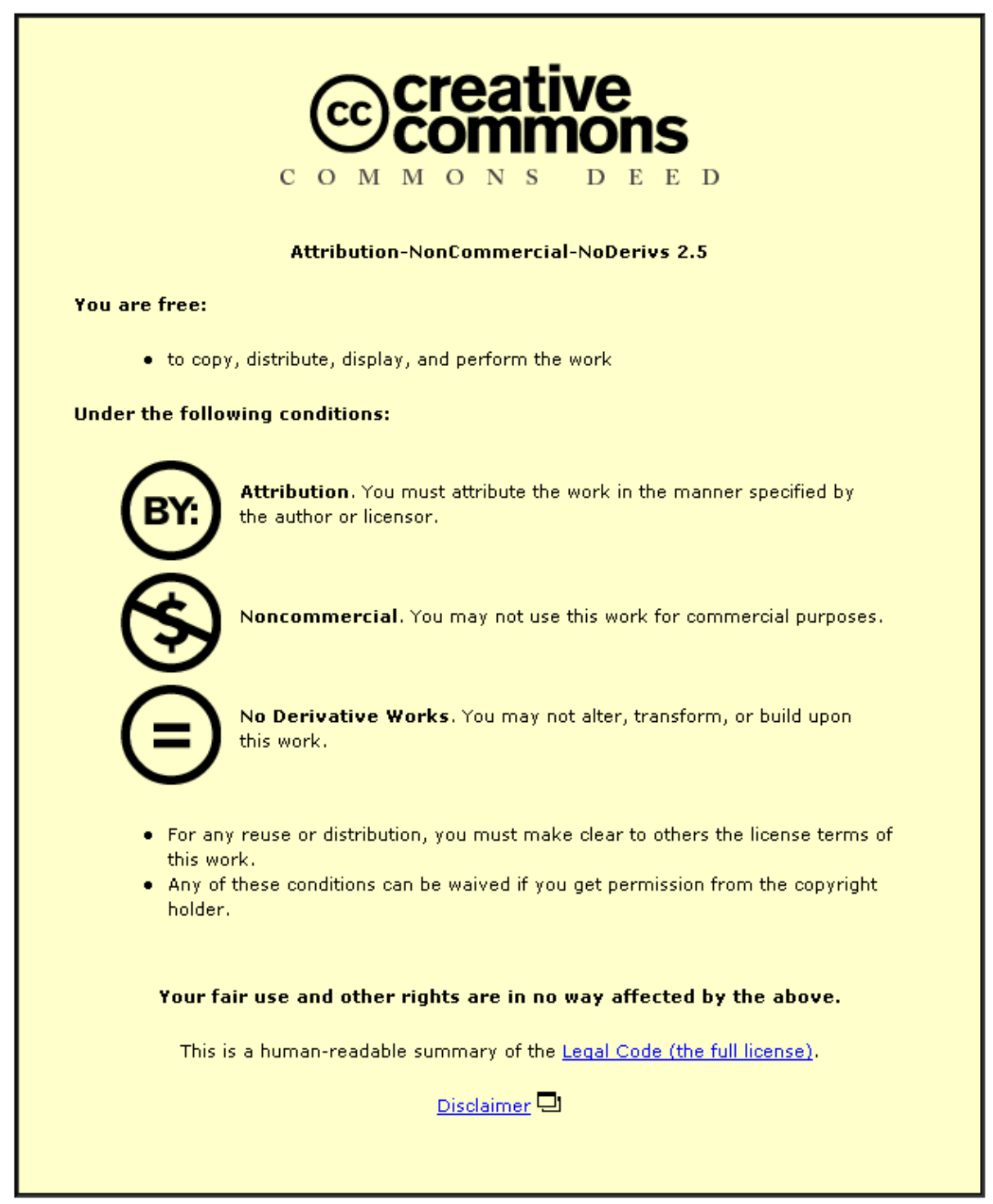

For the full text of this licence, please go to: http://creativecommons.org/licenses/by-nc-nd/2.5/ 


\title{
Eco-Design Tool to support the Use of Renewable Polymers within Packaging Applications
}

\author{
James Colwill, Shahin Rahimifard, Allen Clegg \\ Centre for Sustainable Manufacturing and Reuse/Recycling Technologies (SMART), Wolfson School of Mechanical and \\ Manufacturing Engineering, Loughborough University, UK
}

\begin{abstract}
Bioplastics derived from renewable polymers such as sugars, starches and cellulose, have attracted significant interest from companies looking to reduce their environmental footprint. New production capacity and improved materials have resulted in their increasing adoption for mainstream consumer products packaging. However questions remain regarding their overall environmental benefits and how the maximum environmental gain can be achieved. These uncertainties highlight the need for a decision support tool to aid the packaging design process. This paper examines the issues surrounding bio-derived polymer use and discusses the development of an eco-design tool to assist in their rapid and efficient adoption.
\end{abstract}

Keywords:

Eco-design; Renewable Materials; Biopolymer Packaging

\section{INTRODUCTION}

The annual global production capacity of bio-derived polymers (BDPs) has been forecast to grow annually by 37 percent, reaching 2.33 Million tonnes by 2013 [1]. This rapid growth has been sustained as BDP packaging markets expand from the early adopters producing niche and synergetic items such as organic drinks and whole foods, to global mainstream products and brands such as cola, crisps and chocolate [2]. A key driver of this success has been the desire for environmentally friendly, sustainable packaging and the belief that BDPs meet this requirement. To a large degree this view has been fostered both from the claims made by manufacturers, and the obvious emotional attraction towards a material with a natural, renewable pedigree. More recently this market demand has been further encouraged by various government initiatives which promote and support the procurement of 'bio-based' and 'sustainable' products [3].

Unfortunately, the current level of scientific understanding of the environmental benefits achievable from these materials, particularly post gate (use and end of life stages), is inadequate or simply nonexistent [4]. This is supported by the findings of a review of 25 published LCA reports from the academic and commercial literature, spanning the period between 1997 and 2009, Figure1 [2].

Specific questions, regarding the impact on food production, genetic modification, consistency of supply, technical performance, contamination of conventional polymer waste streams and biodegradability, remain unanswered. Whilst government support for renewable materials is desirable if not essential, caution should be taken to avoid the premature or inappropriate adoption of a particular BDP or technology, which in turn could hinder future development, particularly if the environmental claims are later proven to be false or vacuous.

This paper begins with an overview of the main BDPs used as packaging, their key applications and potential market growth. It then considers the various issues that surround the use of BDPs and identifies the key barriers and drivers to wider and greater adoption. In light of the growing need for sustainable manufacturing,

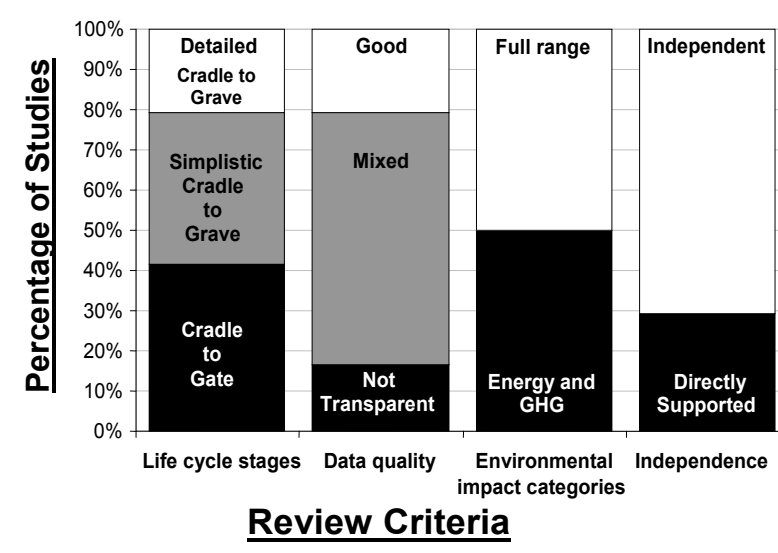

Figure 1: Findings of LCA study against review criteria (2009) [2]. we then consider the range of eco design and decision support tools that are available to industry to assist in the identification, selection, application and assessment of BDP packaging. This study, through an assessment of the key strengths and weaknesses of each tool, aims to identify the key unfulfilled needs in this area and thus establish both the need and the framework for the new eco-design tool. The paper concludes with an overview of this new tool, its proposed structure, and how this will meet the unfulfilled needs of industry.

\section{BIO DERIVED POLYMERS IN PACKAGING}

\subsection{Key BDPs: Their Origins and Evolution}

Whilst a small number of BDPs, such as cellulose film, have maintained a commercial presence in the packaging market, the resurgence in interest of BDPs as a viable alternative to conventional polymers began during the 1990's in response to increasing pressure from both consumers and government to reduce the environmental impact of packaging culminating in the EU directive 94/62/EC on Packaging and Packaging Waste [5]. Whilst the direc- 
tive and subsequent legislation does not promote the use of bio derived materials over conventional ones, it obligates companies to formally consider the environmental aspects of their packaging designs in addition to the commercial and technical ones.

The first generation of BDPs were limited to low technical performance applications, in the past decade a new generation of materials have been developed, capable of being used for processed, long shelf life products such as crisps, cereals, chocolate and beverages. Figure 2 identifies the key BDPs used in packaging and the main source/route to production [5]. As their availability and costs have improved, so their uptake has increased. The most commercially successful of these to date are PolyLactic Acid (PLA) and Bioethylene based PE and PET. Both these materials have been used in full or in part across a wide range of pack formats and processes such as; stretch blow molded bottles, injection molded components, thermoformed trays and flexible films (including high barrier laminated films for coffee and crisps).

\subsection{Packaging Applications Study}

To understand how the application of BDPs for packaging has evolved, an online review of published announcements for new product launches in BDP packaging was undertaken. This included searching the websites and press archives of all the main BDP manufacturers, associated trade press and the key industry bodies, associations and institutes for; the environment, packaging and plastics industries, dating back to 2004 .

It is an expected and an accepted limitation of this review that as a material becomes established, i.e. first generation bio-polymers such as cellulose film and foamed starch chips, it will probably become less noteworthy of comment and so its frequency will decline even if use actually increases. Also, the results recorded launch activity, not ongoing use, and so should not be viewed accumulatively.

When these new introductions are plotted against their launch dates, a picture emerges of a gradual annual growth in use, see Figure 3 lower line. However, this only shows the frequency of product launches and does not consider the individual significance of each new introduction in terms of the BDP used. As it is not possible from these announcements alone to ascertain accurate data with regard to the volume of sales, material use, specific barrier properties, transmission rates etc, a simple weighting factor was applied instead.

The factor used was allocated based on five easily assessable key criteria: Brand awareness, Company size, Launch market size, Potential market size and Application complexity. A weighting factor was applied for the first four criteria of $1 \mathrm{x}$ for local, $3 \mathrm{x}$ for national or $5 x$ for global. For the fifth criteria, application complexity, a weighting of $1 x$ for low complexity, $3 x$ for medium (thermoformed/ laminated), $5 x$ for high complexity (injection molded, blown, high barrier). Once applied the sum total was divided by five to a final value of between 1 and 5 for each application.

When this data is re-plotted with the weighting factor it shows a much sharper growth curve (figure 3 , upper line) particularly during the last two years, which might indicate that BDPs are entering a new accelerated growth phase. This would lead to higher growth than other data has previously suggested, such as BDP production capacity investments [1], which forecast growth by 2020 to reach 3.5 Mt capacity and earlier projections which forecast volumes of between $2.5 \mathrm{Mt}$ and $4.17 \mathrm{Mt}$ by 2020 [6]. In addition, when the two graphs are compared it suggests that in addition to a general increase in use, these new BDPs are gaining wider market acceptance, moving from niche, synergetic applications such as organic, fair-trade and health food products to mainstream, high profile brands.

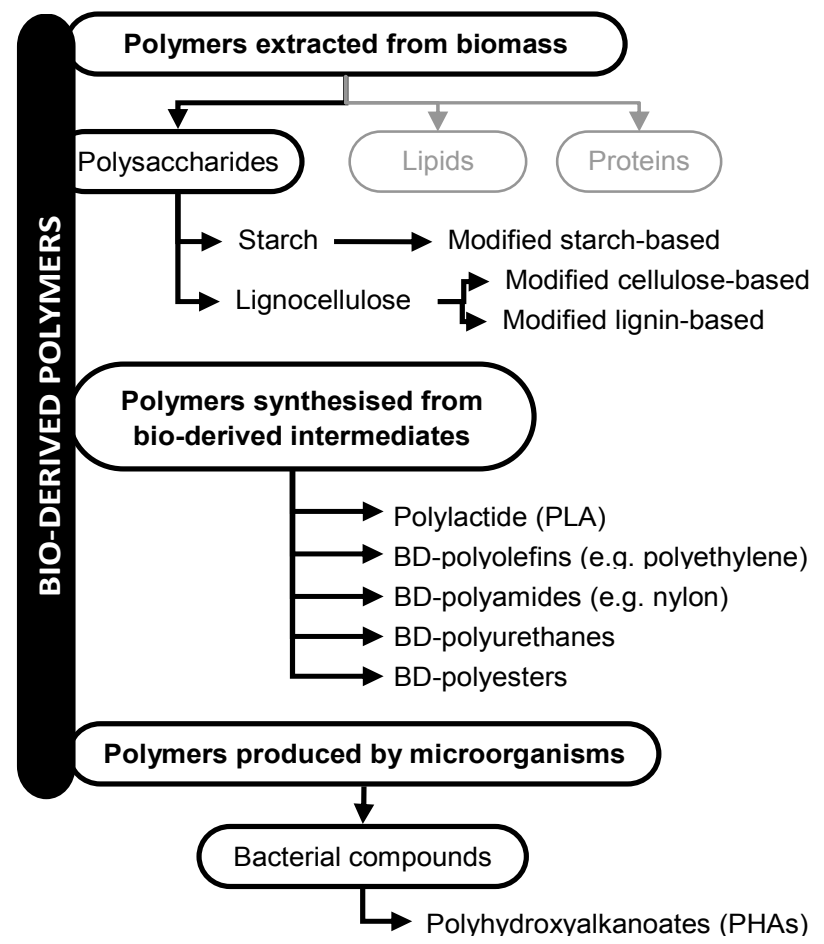

Figure 2: Overview of principal bio-derived polymers (adapted from [6]). Flows in bold indicate routes to the principal BDPs.

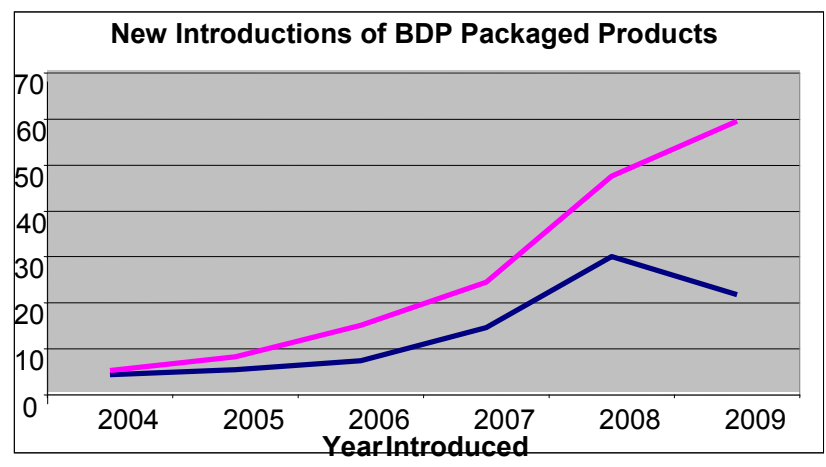

Figure 3: New Introductions of BDPs based on company announcements from Jan 04 to May 09 - Colwill et al [1]

\subsection{Capacity and New Investments}

In anticipation of the future demand, a number of companies have invested in plant for the production of BDP's The annual global production capacity of BDPs, based on company announcements, is now forecast to grow from $0.36 \mathrm{Mt}$ (million metric tonnes) in 2007 to $2.33 \mathrm{Mt}$ in 2013, an annual increase of 37 percent [1]. Figure 4 shows the projected growth in the production capacity of Class $A$ and Class B BDPs. Class A BDPs include PLA, PHA. TPS and cellulose, whilst class B BDP's are those which are identical to conventional polymers apart from the original monomer source, such as PE and PET derived from bio-ethylene.

\section{THE KEY ISSUES TO USING BDPS IN PACKAGING}

\subsection{Drivers and Barriers}

There are a number of factors which to a greater or lesser degree have had or will continue to have an influence on the development, uptake and growth of bio-derived polymers within the packaging 


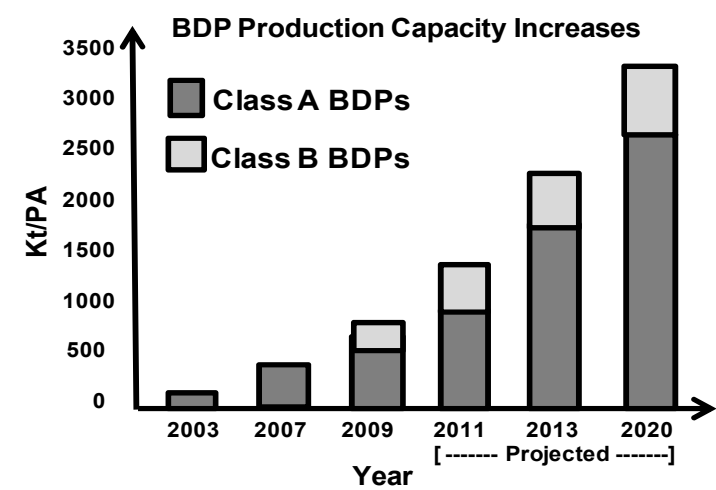

BDP Production capacity Increases

Figure 4: Global production capacity of bio-derived polymers based on company announcements up to May 2009 [2].

sector. The most significant of these are listed in Table 1, however whilst many of these have a foreseeable resolution as technology or commercial advances are made, there are two key issues that in our view will require a much more substantial and collaborative effort to resolve, these are:

- Development of alternative feedstocks to avoid direct competition with food production (materials and land use) in order to provide a sustainable and scalable polymer source.

- Development of new technologies and infrastructure to enable the conservation of this resource and to avoid contamination and disruption of existing conventional polymer recycling.

In terms of positive influences, policy and government initiatives such as the EU's "Lead Market Initiative", the ADEME's "Bioproducts Guidebook for Greener Procurements" and the USA's "Federal Bio-based Products Preferred Procurement Program" have the potential to be a major influence on BDP growth and uptake. The other major driver will be cost and performance parity as the gap between BDPs and conventional plastics narrows.

\subsection{Packaging Design and Development}

The varied and cross departmental responsibilities for packaging functions within a business add yet further complexity to the packaging development process, (Figure 5). Whilst the majority of functions are clearly aligned to a particular hierarchical structure, e.g. Finance and Accounting, Sales and Marketing, Engineering and Production, packaging impacts on almost all aspects of the business and often the control hierarchy will change on a regular basis as a means to adjusting an imbalance caused by that particular departmental bias, (finance, marketing, operations etc). This has often resulted in the packaging function 'ownership' being rotated through different business functions on an almost cyclical basis, Manufacturing, Marketing, Finance/Purchasing etc. One approach some companies have taken is to break the packaging functions into three separate groups as shown in Figure 5.

This allows each function to be more closely aligned with the most appropriate business functions. However this then creates the problem of ensuring that communication and cooperation between the groups maintains the skills and potential of the whole, particularly important in the development of new packaging.

\section{Factors influencing BDP adoption}

The limited availability and increasing cost of fossil resources (oil and gas) and the need to secure National energy supplies.

$\boldsymbol{\Phi}$ Policy and legislation, particularly within the area of climate change, sustainability and economics.

Consumer demand driven by the growing awareness of the need for sustainable management of natural resources.

ص. Other factors include: Organic and green brands, Retailer pressure, anti litter action and increasing environmental problems and severe climate changes.

Higher costs and more complex supply chains, including capacity

(4) limitations and a restricted supplier base.

Technical performance limitations compared to conventional polymers. Lack of clarity and quality of data on the overall environmental impacts.

() Other factors include: Greater recycling of conventional polymers and

Z problems of waste stream contamination by BDPs, Land availability and food production.

Bio-Fuel developments compete for limited feedstock resources but also provide volume, a secure market, and commercial scale.

c Pressure groups can influence public opinion and government policy. However, views are polarised for and against at present.

New technologies such as genetic modification and nano engineering bring huge potential benefits but also huge potential risks. Tend to polarise opinion particularly within an already sensitized and sceptical public.

Table 1: Barriers and Drivers to increased BDP adoption.

It is clear that the decision to adopt BDPs for packaging within an organization will not be restricted to any one group, function or skill set. For the tool to be fully inclusive it needs to engage actors at all levels and stages depending on their abilities and needs. This is true not just within the company but also throughout the wider supply chain and where possible engaging the consumer.

\section{AN OVERVIEW OF CURRENT ECO DESIGN TOOLS}

A study of academic papers and industrial reports was carried out across a range of eco-design tools. This included individual $[7,8]$ as well as multiple $[9,10]$ tool reviews. The main focus was on packaging but general eco-design tools that could be used for packaging design were also considered. The review focused on a number of criteria, four of which have been selected for comparison in Table 2 and Figure 6. These are: Sustainability Considerations (Which of the three key pillars of Sustainability, Environmental, Economic and Social, were considered by the tool), Life Cycle Approach (What life cycle stages were considered), User Guidance (Which of the 5 guidance criteria listed were output to the user) and User Inclusiveness (of the user groups listed, how many would the tool be useful and accessible to).

In all, 40 tools were assessed using a combination of previous design tool studies and individual tool reviews. The main criteria and sub divisions are listed below in Table 2. It is clear that significant interest exists, within a range of industries operating at various stages along the supply chain, in the development of tools for the purpose of improving the environmental design of packaging as well as using renewable materials. 


\section{Packaging Functions}

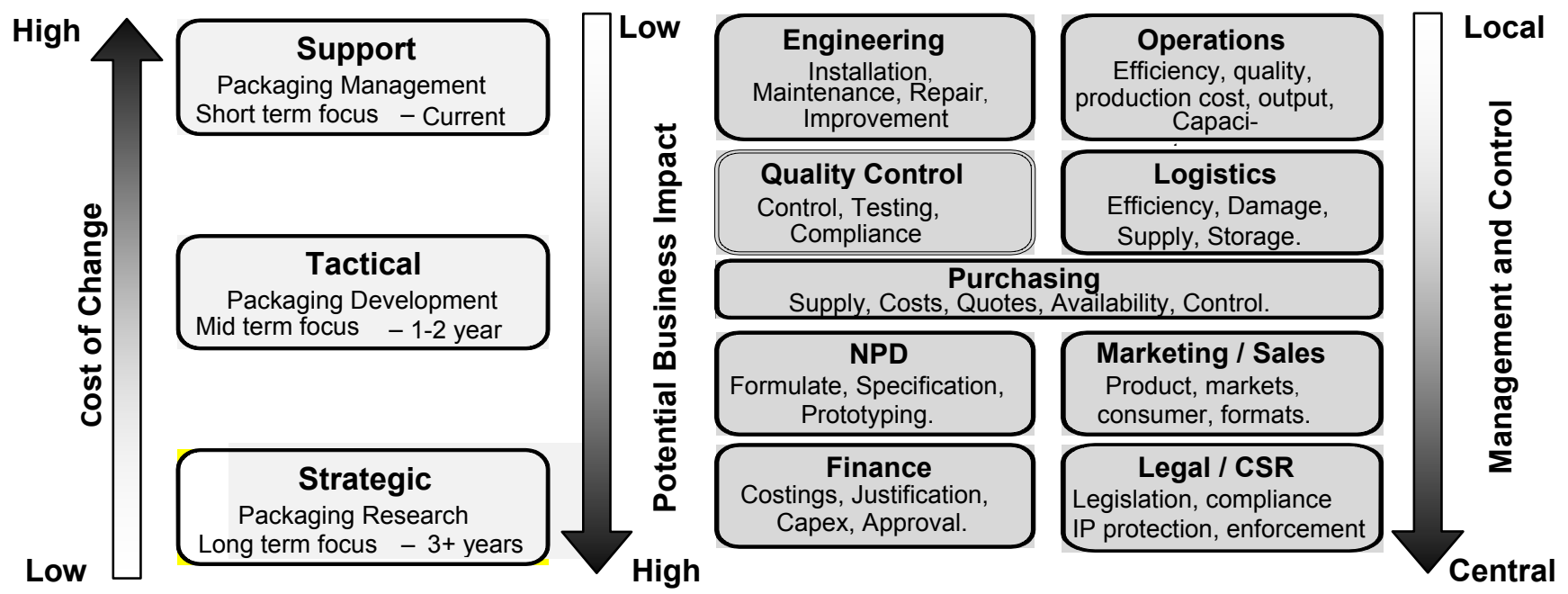

Figure 5: Key functions of a packaging dept and their relation to other key business areas.

\section{FRAMEWORK FOR THE TOOL}

\subsection{Introduction to the tool}

The development of the proposed tool arose from the recognition of the necessity to ensure that the limited capacity of bio-polymers needs to be directed towards applications where the greatest overall environmental benefit can be achieved. It was envisaged that a tool which could help achieve this through the appropriate selection and application of materials within the pack design and development process, would be widely welcomed by industry. [11]. It is also clear that a direct comparison of BDPs with their conventional counterparts would be misleading as to the future potential that could be achieved once the BDP industry and markets mature. The ability of the tool to evaluate the pack based on future potential, as well as current performance, is essential if it is to play a strategic role [12].

\subsection{Key requirements of the tool}

The requirements for the eco-design tool were identified from both a literature review and through industry consultation. Six key requirements are listed in Table 3. The features highlighted in bold are those which are considered to be absent or inadequately provided for in existing tools. These are supported by similar findings in a recent Canadian Government report [10].

\begin{tabular}{|l|c|c|c|c|}
\hline \multicolumn{2}{|c|}{ Assessment Criteria } & & & \\
\hline Sustainability Considerations: & En & En \& Ec & En \& So & En, Ec \& So \\
\cline { 2 - 5 } $\begin{array}{l}\text { Environmental (En), Economic (Ec), Social } \\
\text { (So) }\end{array}$ & 20 & 13 & 6 & 1 \\
\hline \hline $\begin{array}{l}\text { Life Cycle Approach: } \\
\text { Full (C2C), Cradle to Gate (C2G), Gate to } \\
\text { Cradle (G2C), None }\end{array}$ & None & C2G & G2C & C2C \\
\cline { 2 - 5 } & $40.0 \%$ & $15.0 \%$ & $27.5 \%$ & $17.5 \%$ \\
\hline User Guidance: & 1 & 2 & 3 or 4 & all 5 \\
\hline $\begin{array}{l}\text { Descriptive, Selective, Prescriptive, } \\
\text { Assessment, Comparative }\end{array}$ & 21 & 18 & 1 & 0 \\
\hline \hline User Inclusiveness: & $52.5 \%$ & $45.0 \%$ & $2.5 \%$ & $0.0 \%$ \\
\hline $\begin{array}{l}\text { Specialist, Bussiness, Supply Chain (SC), } \\
\text { Supply Chain \& Consumer (SC\&C) }\end{array}$ & 24 & 11 & 5 & 0 \\
\hline
\end{tabular}

Table 2: Results of Ecodesign Tool Study against review criteria.

\begin{tabular}{|l|l|}
\hline \multicolumn{1}{|c|}{ Feature } & \multicolumn{1}{c|}{ Requirements } \\
\hline $\begin{array}{l}\text { Full Life Cycle } \\
\text { Perspective }\end{array}$ & $\begin{array}{l}\text { Should consider performance across the whole life } \\
\text { cycle, cradle to cradle. }\end{array}$ \\
\hline $\begin{array}{l}\text { Sustainable } \\
\text { Focus }\end{array}$ & $\begin{array}{l}\text { The tool should consider all three pillars of sustaina- } \\
\text { bility: Social as well as Environmental and Economic. }\end{array}$ \\
\hline $\begin{array}{l}\text { Strategic and } \\
\text { Tactical }\end{array}$ & $\begin{array}{l}\text { The tool should support strategic decision making } \\
\text { looking at future performance as well as current } \\
\text { properties and performance. }\end{array}$ \\
\hline $\begin{array}{l}\text { Holistic and } \\
\text { Inclusive }\end{array}$ & $\begin{array}{l}\text { Should be usable and provide guidance across the } \\
\text { whole supply chain, including consumers. }\end{array}$ \\
\hline $\begin{array}{l}\text { Total Stage } \\
\text { Support }\end{array}$ & $\begin{array}{l}\text { Should provide support at each stage of the design / } \\
\text { development process through a series of individually } \\
\text { targeted but connected tools. }\end{array}$ \\
\hline Feedback & $\begin{array}{l}\text { Tool should provide feedback which allows progress } \\
\text { to be measured and improved. }\end{array}$ \\
\hline
\end{tabular}

Table 3: Key features, requirements and intended users of the tool.

\subsection{Proposed Structure for the Packaging Eco-Design Tool}

The tool aims to support the decision process at three different levels depending on the expertise of the user, availability of input data and required detail of output data as shown in Figure 6.

This will include; type of application or product to be packaged, selection and use of the BDP material, pack construction, manufacturing process, distribution and retail methods, consumer use and 'end of life' management.

The three separate but interlinked tools, which can be used independently or in combination, are as follows:

\section{EcoD2 Part 1 - Justification Level}

Assesses the potential for including BDP packaging as part of the company's overall packaging / corporate sustainability strategy:

Method: A series of questions, in the form of a decision tree, are asked which highlight the key threats and opportunities, strengths and weakness for the adoption of BDPs by the company, both short and long term.

Result: The results from the questions will give a top level guidance on how the company should proceed. This might include statements such as: 
- BDPs are not compatible with your current business practice and strategy.

- BDPs will provide significant benefits but not within current cost limits.

- BDPs are a viable option for your company, proceed to next level.

\section{EcoD2 Part 2 - Specification Level}

Identify specifically which BDPs will meet the essential and desirable requirements of the specific application regarding technical, commercial and operational feasibility:

Method: A technical relational database of all BDPs commercially available will allow specific requirements to be searched and the suitable polymers to be identified. Each of the key known factors can be entered via a series of blank forms or lists, e.g. Barrier, Strength, Elasticity, Compression, Melt temperature, Process etc.

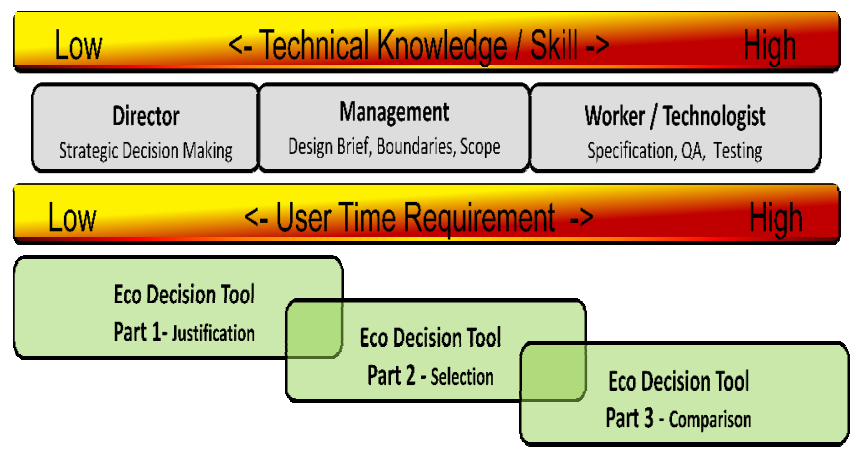

Figure 6: The relationship between User time and skill levels with the three separate Tools parts 1 - 3 .

Result: The results from this stage will be in the form of single datasheets and comparative performance graphs to include:

- Data sheet for each BDP that meets or exceeds entered criteria.

- Multiple BDPs can be plotted against single or multiple criteria.

- Potential future scenarios can be used to give a predicted performance potential.

\section{EcoD2 Part 3 - Comparison Level}

Compares different pack concepts across a range of criteria and supports the final selection process as part of existing new pack development procedures.

Method: Each concept is measured in terms of its material content, material type, performance, size, dimensions, weight and key features. These are input into a program via a menu system which performs the necessary calculations.

Result: The final concepts will be measured in terms of their individual material components, total pack performance, construction costs, cube, environmental footprint etc. The results from this stage will be in the form of single page report that summarises the key benefits, costs and performance of each concept.

\section{CASE STUDY - A BAG FOR ORGANIC SALAD}

The following example illustrates how this proposed eco-design tool might have been used during the decision, design and development process for a possible packaging development project. We created the following scenario as the basis for the case study: A company (UKCM) supplies a leading UK supermarket with pre-washed mixed organic salad. Both the manufacturer and retailer had been meticulous in ensuring that the product meets the highest standards of purity, quality and environmental performance. It was desirable and logical therefore that the packaging should reflect those product values. The category manager of the retailer and the marketing director of the manufacturer/supplier arranged a working meeting to discuss and agree a way forward to achieving this goal. During the meeting the Eco-design Tool (EcoD2) Part 1 was used to investigate whether BDPs might provide a viable packaging solution.

\section{EcoD2 Part 1 - Justification for Using BDP Packaging.}

With only a limited time available a quick answer was required to be derived from information that was readily available to the two "high level' experienced but not technical business people.

Method: The company's Marketing director accessed the tool online to assess the suitability of BDPs as a means to package their product in a 'carbon neutral' way. Following a decision tree based question and answer process, he input top line information about the company, its product and overall aims and objectives, a process that took approximately 10-15 minutes.

Result: The tool provided guidance as to the suitability of BDPs, the main implications of its use and recommended next steps on how the company should proceed:

- Based on the product's brand values, market positioning, premium price, technical/performance requirements and potential end of life disposal options, there is a strong possibility that BDPs could provide a suitable packaging medium for this product

- The BDPs which meet the product requirements and are within a viable geographic range would be Starch, Cellulose or PLA based. Option buttons would be provided which would allow the company to produce a chart comparing specific properties of these 'base' materials on factors such as cost, bio-degradability and technical properties. A list of suppliers could also be generated within a given geographic range.

- The suggested next steps, assuming that the commercial and technical requirements fell within the given range, would be to select and contact the suppliers of these materials initially with a specification / brief to be prepared from the information added to the system so far and to be further populated by the technical and operational staff within the two organizations.

- The specification is sent to the supplier and linked to the tool. The supplier's response is entered into the tool online. This allows comparisons between the different supplier/material options to be compared.

\section{EcoD2 Part 2 - Specification Level}

In order to complete the specification, the technical/packaging manager/technologist identifies specifically which commercially available grades of BDPs from which suppliers meet the technical and performance product requirements. The materials that fulfill these needs are added to the specification.

Method: A technical relational database of all BDPs commercially available allows for specific requirements to be searched and the suitable polymers to be identified. Each of the key known factors can be entered via a series of blank forms or lists. e.g. Barrier, Strength, Elasticity, Compression, Melt temperature, Process etc.

Result: The results from this stage will be in the form of datasheets and comparative performance graphs. In addition the qualifying materials and supplier information can be transferred from the database to the specification sheet for transmission to the supplier. This can also be used to automatically request quotes, technical data and trial sample materials.

\section{EcoD2 Part 3 - Comparison Level}

Following initial trials of the different materials, the comparison tool is used by the designer to compare the different pack concepts 
across a range of criteria and to use this data to support the final selection process as part of in-house new pack development procedures. The outputs of this information can be stored and made available to consumers via the tool or other medium such as the retailer's website.

Method: Each concept is measured in terms of its material content, material type, performance, size, dimensions, weight and key features. These are input into a program via a menu system which performs the necessary calculations.

Result: The final concepts will be measured in terms of their individual material components, total pack performance, construction costs, cube, environmental footprint etc. The results from this stage will be in the form of single page report that summarises the key benefits: environmental, commercial, social and physical performance for each concept. In addition, comparative charts and graphs can be produced for each of these key criteria.

\section{SUMMARY AND CONCLUDING DISCUSSIONS}

Whilst the growth and development of bio-derived polymers has continued to gain momentum over the past few years, there is a clear danger that this could stall if confusion regarding their overall environmental impact is not removed. A number of methods for categorizing BDPs have been suggested, such as by feedstock type or production method, however in terms of application and end of life management there are two main divisions: Class A, unconventional polymers extracted or synthesized from renewable feedstock but not compatible with conventional plastics and Class B, conventional polymers synthesized from bio-ethylene e.g. polyethylene and PET. It is these former class A bio-polymers, such as PLA, Cellulose, PHA and TPS, that require further investigation in this area in order for them to achieve their environmental potential.

In parallel with the growth of BDPs, there has been the pressure on companies to reduce their manufacturing environmental footprint particularly that associated with their packaging. To-date this has focused primarily on waste reduction and recycling and in some instances materials substitution, such as replacement of PVC with PET. As a result, a number of guides and tools have been developed to assist companies in achieving these goals; including Life Cycle Assessment, Retailer Scorecards and Green Design Guides. However these guides tend to be limited in the guidance that they give, strategic and early design stage use, the range of impacts measured, the cost and complexity of use and/or the over simplification of the results. In particular for BDPs, it is important to consider the likely future impacts as technologies, costs and methods advance.

As packaging is a multi disciplinary function that extends across the majority of traditional business departmental boundaries, it is essential that this tool provides a mechanism for a wide range of users with different skills and requirements to input into and benefit from its use. Furthermore, the use of the tool should extend beyond the traditional business operations and be available to the whole supply chain. In particular the information should be available to the consumer to enable them to make informed choices about the products they buy which in turn will drive further environmental investment and development by industry.

It is clear therefore that a holistic approach is needed to eco packaging design if the future challenges of sustainability are to be achieved. It is also clear that better guidance at both the strategic and tactical level on the selection and use of bio-derived polymers in packaging applications is required by industry to avoid 'green wash' and ensure the greatest environmental, sustainable and ecological return is achieved from this renewable but ultimately finite resource. The eco-design decision tool which we are develop- ing for packaging will be a significant step towards achieving these goals.

\section{REFERENCES}

[1] Shen, L., Haufe, J., Patel, M. (2009): Product overview and market projection of emerging bio-based plastics, PRO-BIP 2009, Group Science, Technology and Society (STS), Copernicus Institute for Sustainable Development and Innovation Utrecht University.

[2] Colwill, J et al (2009): Opportunities for bio-polymer resource conservation through closed loop recycling, in: Proceedings of GPEC 2010 on Sustainability \& Recycling: Raising the Bar in Today's Economy, Orlando, Florida USA.

[3] Skibar W., Grogan G., Pitts M., Higson A., (2009): Analysis of the UK Capabilities in Industrial Biotechnology in Relation to the Rest of the World, a sector assessment for the (IB-IGT) prepared by Bioscience for Business KTN, Chemistry Innovation KTN and the NNFCC.

[4] Song, B., Lee, R.J., Lu, W.F. (2010): A Study on the EcoPerformance of Plastics in the Injection Molding Process, Proceedings of 17th CIRP International Conference on Life Cycle Engineering, Hefei, China, 180-185.

[5] European Parliament and Council Directive 94/62/EC of 20 December 1994 on packaging and packaging waste, Accessed online at http://eurlex.europa.eu/LexUriServ/LexUriServ.do?uri=CONSLEG:1 994L0062:20090420:EN:PDF

[6] Quieroz A.U.B. and Collares-Quieroz F.P. (2009): Innovation and Industrial Trends in Bioplastics, Journal of Macromolecular Science, Part C: Polymer Reviews 49 65-78.

[7] Crank, M., Patel, M.K., Marscheider-Weidemann, F., Schleich, J., Hüsing, B., Angerer,G. (2005): Techno-economic feasibility of large-scale production of bio-based polymers in Europe. Report No. EUR No: 22103 EN, Catalogue (OPOCE): LFNA-22103-EN-C.

[8] Walmart (2009), Sustainable Product Index: Fact Sheet, accessed online at www.walmartstores.com/Sustainability/9292.aspx, 5th October 2009.

[9] Huo, L., Saito, K. (2007): Concept Identification and Implementation of Sustainable Packaging Systems, Journal of Packaging Science Technology, Vol. 16 No. 4 P269-281.

[10] Five Winds International (2008): Inventory of Sustainable Packaging Initiatives and Proposed Approach to Develop Sustainable Packaging Guidelines, prepared for the extended Producer Responsibility Task Group CCME (Canadian Council of Ministers of the Environment).

[11] Novkov, S. (2008): Sustainability Management of Industrial Enterprises Advanced Concepts, Techniques and Tools, presented at the 5th International Scientific Conference 'Business and Management' Vilnius, Lithuania, 16-17 May 2008.

[12] Sonneveld, K., James, K., Fitzpatrick, L., Lewis, H., (2005): Sustainable Packaging: How do we Define and Measure It?, presented at the 22nd IAPRI, Symposium, Campinas, Brazil, 22-24 May 2005 